TRANSACTIONS OF THE

AMERICAN MATHEMATICAL SOCIETY

Volume 361, Number 4, April 2009, Pages 2163-2179

S 0002-9947(08)04607-2

Article electronically published on November 14, 2008

\title{
WEIGHTED POLYNOMIALS AND WEIGHTED PLURIPOTENTIAL THEORY
}

\author{
THOMAS BLOOM
}

\begin{abstract}
Let $E$ be a compact subset of $\mathbb{C}^{N}$ and $w \geq 0$ an admissible weight function on $E$. To $(E, w)$ we associate a canonical circular set $Z \subset \mathbb{C}^{N+1}$. We obtain precise relations between the weighted pluricomplex Green function and weighted equilibrium measure of $(E, w)$ and the pluricomplex Green function and equilibrium measure of $Z$. These results, combined with an appropriate form of the Bernstein-Markov inequality, are used to obtain asymptotic formulas for the leading coefficients of orthonormal polynomials with respect to certain exponentially decreasing weights in $\mathbb{R}^{N}$.
\end{abstract}

\section{INTRODUCTION}

An admissible weight on a compact set $E \subset \mathbb{C}^{N}$ is a u.s.c. function $w \geq 0$ which is strictly positive on a non-pluripolar subset of $E$. Associated to $(E, w)$ is a weighted pluripotential theory involving weighted polynomials, i.e., functions of the form $w^{d} p$ where $p$ is a polynomial of degree $\leq d$, a weighted pluricomplex Green function $V_{E, Q}$ and a weighted equilibrium measure $\mu_{e q}(E, w)$. The definitions of these concepts are given in section 1 .

In the one-dimensional case $(N=1)$ the book of Saff and Totik [SaTo] has many basic results. In the one-dimensional case, weighted polynomials arise in diverse problems: approximation theory, orthogonal polynomials, random matrices, statistical physics. For an example of recent developments see [Dei].

In the higher-dimensional case, weighted pluripotential theory was used in BL2 to show that two polynomially convex compact sets $E \subset F \subset \mathbb{C}^{N}$ differ by a pluripolar set if and only if they have the same transfinite diameter, the main procedure being an inductive step from circular compact sets to a weighted problem in one less variable.

In this paper we further develop the relation between weighted pluripotential theory on a compact set $E \subset \mathbb{C}^{N}$ with admissible (see (1.10)) weight $w$ and the potential theory of a canonically associated circular set $Z \subset \mathbb{C}^{N+1}$ (defined in (2.1)).

We show that $H_{Z}$, the homogeneous pluricomplex Green function of $Z$, and $\mu_{e q}(Z)$, the equilibrium measure of $Z$, are related to the weighted pluricomplex Green function and the weighted equilibrium measure of $E$ with weight $w$.

The main results are:

Theorem 2.1. $H_{Z}=\left(V_{E, Q}\right) \circ L+\log |t|$ for $t \neq 0$.

Received by the editors September 15, 2006 and, in revised form, May 30, 2007.

2000 Mathematics Subject Classification. Primary 32U20, 32U35.

The author was supported by an NSERC of Canada Grant.

(C)2008 American Mathematical Society 
Theorem 2.2. $L_{*}\left(\frac{1}{2 \pi} \mu_{e q}(Z)\right)=\mu_{e q}(E, w)$.

Here we use the notation $(t, z)$ for a point in $\mathbb{C}^{N+1}$ where $t \in \mathbb{C}, z \in \mathbb{C}^{N}$ and $L:\left\{\mathbb{C}^{N+1} \backslash\{(t, z) \mid t=0\}\right\} \rightarrow \mathbb{C}^{N}$ is $L(t, z):=\frac{z}{t}$ (see (2.4)). $L_{*}$ is the push-forward of measures under $L$.

Special cases of the above results may be found in the paper of DeMarco DeM]. In particular Theorem 2.1 generalizes examples of Section 4 of [DeM] and Theorem 2.2 generalizes Lemma 2.3 of [DeM].

The advantage of considering weighted pluripotential theory is that (up to a limiting procedure described in section 5 of this paper) the potential theory of a general compact circular set in $(N+1)$ variables may be reduced to the weighted case in $N$ variables.

In section 3 we consider the Bernstein-Markov (B-M) inequality (for the definition see (3.1)). This inequality may be used to relate asymptotics of orthonormal polynomials with respect to a measure $\mu$ on $E$ to potential-theoretic invariants of $E$. We introduce a weighted version of the B-M inequality depending on $E$, the weight $w$ and the measure $\mu$ (see (3.2)). We show (Theorem 3.1) that the weighted B-M inequality holds on $E$ with weight $w$ and measure $\mu$ if and only if the B-M inequality holds for an associated measure on $Z$. Then we give the following cases where the weighted B-M inequality holds.

Corollary 3.1. The weighted $B-M$ inequality holds for $E$ locally regular and $w, a$ continuous, admissible weight $\left(E, w, \mu_{e q}(E, w)\right)$.

Theorem 3.2. The weighted $B-M$ inequality holds for $(E, w, \sigma)$, where $E \subset \mathbb{R}^{N}$ and $(E, \sigma)$ satisfies the $B$-M inequality and $w$ is continuous with $\inf _{z \in E} w>0$.

In section 4 we obtain asymptotics for the leading coefficients of polynomials on $\mathbb{R}^{N}$ orthonormal with respect to a measure of the form $\exp (-H(x)) d x$ where $H$ is non-negative and homogeneous of $\operatorname{deg} \gamma>0$ (see (4.11)). Such a measure is a multivariable version of the Freud weights whose study was a major motivation in the development of weighted potential theory in one variable (see [SaTo]).

Specifically, after applying the Gram-Schmidt procedure to the monomials, ordered via a lexicographic ordering on their exponents, we obtain for every multiindex $\alpha \in \mathbb{N}^{N}$ an orthonomal polynomial $p_{\alpha}(x)$, which is a linear combination of monomials with multi-index exponents $\leq \alpha$ and with leading term denoted $a_{\alpha} x^{\alpha}$, where $a_{\alpha}>0$. In the multivariable case $(N>1)$ we cannot expect that the full sequence of leading coefficients will have asymptotic limits; simple examples show that this in not so.

We consider sequences of multi-indices $\{\alpha(j)\}$ satisfying (see (4.4)) $\lim _{j}|\alpha(j)|=$ $+\infty$ and $\lim _{j} \frac{\alpha(j)}{|\alpha(j)|}=\theta=\left(\theta_{1}, \cdots, \theta_{N}\right)$ where each $\theta_{i}>0$. We prove

\section{Theorem 4.2.}

$$
\lim _{j \rightarrow \infty}\left(a_{\alpha(j)}^{\frac{1}{\alpha(j) \mid}}\right)|\alpha(j)|^{\frac{1}{\gamma}}=\frac{1}{\tau^{w}\left(S_{w}, \theta\right)},
$$

where $\tau^{w}\left(S_{w}, \theta\right)$ is an appropriate directional Tchebyshev constant.

The general approach to Theorem 4.2 is similar to the one-variable approach in the case of Freud weights [SaTo]. We scale the problem to obtain an equivalent problem involving weighted polynomials. The needed asymptotics for the 
leading coefficients of those weighted polynomials are given in Theorem 4.1. The "unweighted" version of Theorem 4.1 was given in ([B2, Proposition 3.4]).

\section{Preliminaries}

Let $E$ be a subset of $\mathbb{C}^{N}$. The pluricomplex Green function of $E$ is defined by

$$
V_{E}(z):=\sup \{u(z) \mid u \in \mathcal{L}, u \leq 0 \text { on } E\}
$$

where

$$
\mathcal{L}=\left\{u \mid u \text { is plurisubharmonic (PSH) on } \mathbb{C}^{N}, u(z) \leq \log ^{+}|z|+C\right\}
$$

is the Lelong class of PSH functions of logarithmic growth. (We use the notation

$$
\left.|z|:=\left(\sum_{i=1}^{N}\left|z_{i}\right|^{2}\right)^{1 / 2} \text { for } z=\left(z_{1}, \cdots, z_{N}\right) \in \mathbb{C}^{N} .\right)
$$

A set $E \subset \mathbb{C}^{N}$ is said to be pluripolar if for all points $a \in E$, there is a neighborhood $U$ of $a$ and a function $v$ which is PSH on $U$ such that $E \cap U \subset\{z \in U \mid v(z)=$ $-\infty\}$. A property of a set $E$ is said to hold quasi-everywhere (q.e.) if there is a pluripolar set $P \subset E$ and the property holds at all points of $E \backslash P$.

For $G$ an open subset of $\mathbb{C}^{N}$ and $f$ a real-valued function on $G$, we let $f^{*}$ denote its uppersemicontinuous (u.s.c.) regularization, defined by

$$
f^{*}(z)=\varlimsup_{\xi \rightarrow z} f(\xi) \quad \text { for } z \in G .
$$

$V_{E}^{*} \in \mathcal{L}$ if and only if $E$ is non-pluripolar $[\mathrm{K}$. For $E$ non-pluripolar, the equilibrium measure of $E$ is defined by

$$
\mu_{e q}=\mu_{e q}(E):=\left(d d^{c} V_{E}^{*}\right)^{N},
$$

where $\left(d d^{c}\right)^{N}$ is the complex Monge-Ampère operator. $\mu_{e q}$ is a positive Borel measure of total mass $(2 \pi)^{N}$ and with $\operatorname{supp}\left(\mu_{e q}\right) \subset \bar{E}[\mathbf{K}$.

In the case that $E$ is a compact set, it is a result of Siciak and Zaharyuta ( $\mathrm{K}$, Theorem 5.1.7]) that

$$
V_{E}(z)=\log \phi_{E}(z)
$$

where

$\phi_{E}(z)=\sup \left\{|p(z)|^{\frac{1}{\operatorname{deg}(p)}} \mid p\right.$ is a holomorphic polynomial, $\operatorname{deg}(p) \geq 1$ and $\left.\|p\|_{E} \leq 1\right\}$.

It follows that $V_{E}(z)=V_{\hat{E}}(z)$, where $\hat{E}$ denotes the polynomially convex hull of $E$.

We will also use the class $\mathcal{H}$ of logarithmically homogeneous PSH functions on $\mathbb{C}^{N}$ defined by

$$
\mathcal{H}:=\left\{u \in \mathcal{L}|u(\xi z)=u(z)+\log | \xi \mid \text { for all } z \in \mathbb{C}^{N}, \xi \in \mathbb{C}\right\} .
$$

For $E$ a bounded set in $\mathbb{C}^{n}$, we define its homogeneous pluricomplex Green function

$$
H_{E}(z):=\sup \{u(z) \mid u \in \mathcal{H}, u \leq 0 \text { on } E\} .
$$

For $E$ compact (see [Si2]) we have

$$
H_{E}(z)=\log \psi_{E}(z)
$$


where

$$
\begin{aligned}
& \psi_{E}(z)=\sup \left\{|p(z)|^{\frac{1}{\operatorname{deg}(p)}} \mid p\right. \text { is a homogeneous holomorphic polynomial, } \\
& \left.\qquad \operatorname{deg} p \geq 1 \text { and }\|p\|_{E} \leq 1\right\} .
\end{aligned}
$$

An admissible weight function on a closed subset $E \subset \mathbb{C}^{N}$ is a real-valued function $w$ on $E$ satisfying

i) $w \geq 0$.

ii) $w$ is u.s.c. (uppersemicontinuous).

iii) $\{z \in E \mid w(z)>0\}$ is non-pluripolar.

iv) If $E$ is unbounded, then $\lim _{|z| \rightarrow+\infty}|z| w(z)=0$ for $z \in E$.

In particular, if $E$ admits an admissible weight function, then $E$ itself is nonpluripolar.

There is a "weighted" version of the pluricomplex Green function (see [Si1, [SaTo, appendix B]) defined as follows: let

$$
Q:=-\log w .
$$

Then $Q$ is lowersemicontinuous (l.s.c.) on $E$. The weighted pluricomplex Green function of $E$ with admissible weight $w$ is defined by

$$
V_{E, Q}:=\sup \{u(z) \mid u \in \mathcal{L}, u \leq Q \text { on } E\} .
$$

$V_{E, Q}^{*} \in \mathcal{L}($ see $[$ SaTo, appendix B]).

The weighted equilibrium measure of $E$ is defined by

$$
\mu_{e q}(E, w):=\left(d d^{c} V_{E, Q}^{*}\right)^{N} .
$$

It is a positive Borel measure with compact support $\operatorname{supp}\left(\mu_{e q}(E, w)\right) \subset \bar{E}$ and total mass $(2 \pi)^{N}$.

A weighted polynomial on $E$ is defined to be a function of the form $w^{d} p$, where $d$ is an integer $\geq 0$ and $p$ is a holomorphic polynomial of degree $\leq d$. Note that if $\left\|w^{d} p\right\|_{E} \leq 1$, then $\frac{1}{d} \log |p(z)| \leq Q(z)$ on $E$ and since $\frac{1}{d} \log |p(z)| \in \mathcal{L}$ we have $\frac{1}{d} \log |p(z)| \leq V_{E, Q}(z)$ for all $z \in \mathbb{C}^{N}$.

It is known (see [Si1] or [SaTo, appendix B]) that

$$
V_{E, Q}(z)=\log \phi_{E, Q}(z)
$$

where

$\phi_{E, Q}(z)=\sup \left\{|p(z)|^{\frac{1}{d}} \mid\left\|w^{d} p\right\|_{E} \leq 1, \operatorname{deg} p \geq 1\right.$ and $w^{d} p$ is a weighted polynomial $\}$.

A set $E$ is defined to be regular if $V_{E}$ is continuous on $\mathbb{C}^{N}$. A set $E$ is defined ([Si1]) to be locally regular at a point $a \in \bar{E}$ if for each $r>0, V_{E \cap B(a, r)}$ is continuous at $a$. Here $B(a, r):=\left\{z \in \mathbb{C}^{N}|| z-a \mid \leq r\right\}$ denotes the ball with center $a$, radius $r$. It is sufficient, for $E$ to be locally regular at $a$, that $V_{E \cap B(a, r)}$ be continuous at $a$ for all $r>0$ sufficiently small.

$E$ is said to be locally regular if it is locally regular at each point of $\bar{E}$.

For $E$ compact and locally regular and $w$ a continuous admissible weight function on $E$, then $V_{E, Q}$ is continuous [Si1. 
For $u \in \mathcal{L}$ we define its Robin function $\rho_{u}$ by

$$
\rho_{u}(z):=\varlimsup_{\substack{|s| \rightarrow+\infty \\ s \in \mathbb{C}}} u(s z)-\log |s| .
$$

It is known (see $[\underline{\operatorname{Si3}}]$ ) that $\rho(z) \in \mathcal{H}$.

\section{EQUiLIBRIUM MEASURES}

Let $E$ be a compact set in $\mathbb{C}^{N}$ and $w$ an admissible weight function on $E$. We associate the set $Z=Z(E, w) \subset \mathbb{C}^{N+1}$ defined as follows:

$$
Z:=\left\{\left(t, \lambda_{1} t, \cdots, \lambda_{N} t\right) \in \mathbb{C}^{N+1} \mid \lambda=\left(\lambda_{1}, \cdots, \lambda_{N}\right) \in E, t \in \mathbb{C} \text { and }|t|=w(\lambda)\right\} .
$$

We will relate the weighted potential theory on $E$ with weight $w$ to the potential theory on $Z$. We will use the notation $(t, z)$ for a point in $\mathbb{C}^{N+1}$ where $t \in \mathbb{C}$ and $z \in \mathbb{C}^{N}$. For $\lambda \in \mathbb{C}^{N}$, we denote by $C_{\lambda}$ the complex line in $\mathbb{C}^{N+1}$ given by

$$
C_{\lambda}:=\left\{(t, z) \in \mathbb{C}^{N+1} \mid z=t \lambda, t \in \mathbb{C}\right\} .
$$

$Z$ is a circular set; i.e., if $(t, z) \in Z$, then $\left(e^{i \theta} t, e^{i \theta} z\right) \in Z$ for all $\theta \in[0,2 \pi) . \bar{Z}$ (the closure of $Z$ ) is compact and circular.

$Z$ is non-pluripolar since $E$ is non-pluripolar ( BL2, Lemma 6.1]). Note that for $w \equiv 1$ (the "unweighted" case), then $Z=\{|t|=1\} \times E$.

Since $Z \subset \bigcup_{\lambda \in E} C_{\lambda}$ the same is true for $\bar{Z}$ and so $\bar{Z} \cap\left\{(t, z) \in \mathbb{C}^{N+1} \mid t=0\right\}$ is either empty (if $w$ is bounded below, on $E$, by a positive constant) or else consists only of the origin.

Proposition 2.1. $H_{Z}=H_{\bar{Z}}$ and $V_{Z}=V_{\bar{Z}}$.

Proof. To prove the first statement it suffices to show that if $u \in \mathcal{H}\left(\mathbb{C} \times \mathbb{C}^{N}\right), u \leq 0$ on $Z$, then $u \leq 0$ on $\bar{Z}$. But, since $w$ is u.s.c. we have, for $\lambda \in E$ :

$$
\bar{Z} \cap C_{\lambda} \subset\left\{(t, z) \in C_{\lambda}|| t \mid \leq w(\lambda)\right\} .
$$

Applying the maximum principle to the subharmonic function $t \rightarrow u\left(t, \lambda_{1} t, \cdots, \lambda_{N} t\right)$ we have $u \leq 0$ on $\bar{Z}$.

The second statement follows similarly.

For a set $Z(E, w)$ we let

$$
Z(D):=\left\{(t, z) \in \mathbb{C}^{N+1} \mid z=\lambda t, \lambda \in E \text { and }|t| \leq w(\lambda)\right\} .
$$

We note that $Z \subset \bar{Z} \subset Z(D) \subset \hat{\bar{Z}}$. Using Proposition 2.1 and ([K, Theorem 5.1.7]) we have $V_{Z}=V_{\bar{Z}}=V_{Z(D)}=V_{\overline{\bar{Z}}}$. Also $H_{Z}=H_{\bar{Z}}=H_{Z(D)}=H_{\overline{\bar{Z}}}$.

Proposition 2.2. $V_{Z}=\operatorname{Max}\left(0, H_{Z}\right)$.

The statement follows from Proposition 2.1 and ([Si1, Proposition 5.6]).

Proposition 2.3. $\mu_{e q}(Z)$ has compact support in $\mathbb{C}^{N+1}-\{t=0\}$.

Proof. Since $H_{Z}^{*} \in \mathcal{H}$ and $H_{Z}^{*}(0)=-\infty$, then $H_{Z}^{*}<0$ in a neighborhood of the origin (estimates on the size of that neighborhood, known as the Sibony-Wong inequality, can be found in ([A], $\mathrm{Si2}])$ ).

Thus, the origin is an interior point of the polynomial hull $\hat{\bar{Z}}$. But $\mu_{e q}(Z)$ places no mass on the interior of $\hat{\bar{Z}}$, so the result follows. 
Let $L$ denote the mapping $L: \mathbb{C}^{N+1} \backslash\{t=0\} \rightarrow \mathbb{C}^{N}$ given by

$$
L(t, z)=\frac{z}{t}:=\lambda \in \mathbb{C}^{N} .
$$

If we consider $\mathbb{P}^{N}$ (complex projective $N$-space) as the space of lines through the origin in $\mathbb{C}^{N+1}$, then $L$ gives one of the standard coordinate charts for $\mathbb{P}^{N}$. Note that $L\left(C_{\lambda}\right)=\lambda$.

We recall the " $H$-principle" of Siciak [Si3]. There is a natural $1-1$ correspondence between $\mathcal{H}\left(\mathbb{C}^{N+1}\right)$ and $\mathcal{L}\left(\mathbb{C}^{N}\right)$ as follows: To $\tilde{u}(t, z) \in \mathcal{H}\left(\mathbb{C} \times \mathbb{C}^{N}\right)$ associate

$$
u(z):=\tilde{u}(1, z) .
$$

Then $u \in \mathcal{L}\left(\mathbb{C}^{N}\right)$. Conversely, given $u \in \mathcal{L}\left(\mathbb{C}^{N}\right)$ we let

$$
\tilde{u}(t, z):=u\left(\frac{z}{t}\right)+\log |t|=L^{*} u(\lambda)+\log |t| \text { for } t \neq 0
$$

and

$$
\tilde{u}(0, z)=\varlimsup_{\substack{|s| \rightarrow+\infty \\ s \in \mathbb{C}}} u(s z)-\log |s|=\rho_{u}(z) .
$$

Then $\tilde{u} \in \mathcal{H}\left(\mathbb{C} \times \mathbb{C}^{N}\right)$.

Furthermore, let $P_{d}(t, z)$ be a homogeneous polynomial of degree $d$ on $\mathbb{C} \times \mathbb{C}^{N}$. Then $\frac{1}{d} \log \left|P_{d}(t, z)\right| \in \mathcal{H}\left(\mathbb{C} \times \mathbb{C}^{N}\right)$ and the associated element of $\mathcal{L}\left(\mathbb{C}^{N}\right)$ via $(2.5)$ is $\frac{1}{d} \log \left|P_{d}(1, z)\right| . P_{d}(1, z)$ is, of course, a polynomial in $z$ of degree $\leq d$.

Conversely, given a polynomial $G_{d}(z)$ in $z$ of degree $\leq d$, then $\frac{1}{d} \log \left|G_{d}(z)\right| \in$ $\mathcal{L}\left(\mathbb{C}^{N}\right)$. The associated (via (2.6)) function in $\mathcal{H}\left(\mathbb{C} \times \mathbb{C}^{N}\right)$ is $\frac{1}{d} \log \left|t^{d} G_{d}(z / t)\right|$. Note that $t^{d} G_{d}\left(\frac{z}{t}\right)$ is a homogeneous polynomial on $\mathbb{C}^{N+1}$ of degree $d$ in $(t, z)$. We use the notation:

$$
P_{d}(t, z):=t^{d} G_{d}(z / t)
$$

Given a weighted polynomial $w^{d} G_{d}(\lambda)$ on $E$ we can relate its norm on $E$ with the norm of the associated polynomial $P_{d}(t, z)$ on $Z$ (or equivalently, $\bar{Z}$ ). Specifically, we have

Lemma 2.1. $\left\|w^{d} G_{d}\right\|_{E}=\left\|P_{d}(t, z)\right\|_{Z}$.

Proof. For $(t, z) \in Z \cap C_{\lambda}$ and $\lambda \in E$, then

$$
\begin{gathered}
P_{d}(t, z)=t^{d} P_{d}(1, z / t)=t^{d} G_{d}(\lambda), \text { so } \\
\left|P_{d}(t, z)\right|=|t|^{d}\left|P_{d}(1, z / t)\right|=w(\lambda)^{d}\left|G_{d}(\lambda)\right| .
\end{gathered}
$$

The result follows.

Theorem 2.1 below gives the relation between the weighted pluricomplex Green function on $E$ and the homogeneous pluricomplex Green function of $Z$.

Theorem 2.1. $H_{Z}(t, z)=L^{*}\left(V_{E, Q}\right)+\log |t|$ for $t \neq 0$.

Proof. Let $\tilde{u}(t, z) \in \mathcal{H}\left(\mathbb{C} \times \mathbb{C}^{N}\right)$ and suppose $\tilde{u} \leq 0$ on $Z$. Now $\tilde{u}\left(t, \lambda_{1} t, \cdots, \lambda_{N} t\right)=$ $\tilde{u}(1, \lambda)+\log |t|$, so for $\lambda \in E$, we have $\log |t|+\tilde{u}(1, \lambda) \leq 0$ on $C_{\lambda} \cap Z$. Thus,

$$
u(\lambda) \leq-\log |t|=Q(\lambda) \text { on } C_{\lambda} \cap Z .
$$

Hence $u \leq V_{E, Q}$ and so, using (2.7),

$$
\tilde{u}(t, z) \leq L^{*}\left(V_{E, Q}\right)+\log |t| \text { for } t \neq 0 .
$$


Taking the pointwise sup in $(t, z)$ over all such $\tilde{u}$ we have

$$
H_{Z} \leq L^{*}\left(V_{E, Q}\right)+\log |t| \text { for } t \neq 0 .
$$

It remains to prove the reverse inequality. Given $u \in \mathcal{L}\left(\mathbb{C}^{N}\right)$ with $u \leq Q$ on $E$, then for $(t, z) \in C_{\lambda} \cap Z$, using (2.6), we have $\tilde{u}(t, z)=\log |t|+u(\bar{\lambda}) \leq$ $\log |t|-\log w(\lambda) \leq 0$. Hence $\tilde{u}(t, z) \leq 0$ on $Z$ and $\tilde{u}(t, z) \leq H_{Z}$ on $\mathbb{C} \times \mathbb{C}^{N}$. That is,

$$
L^{*}(u)+\log |t| \leq H_{Z} \text { for } t \neq 0 .
$$

Taking the pointwise sup over all such $u$ gives the reverse inequality to (2.13).

Corollary 2.1. $H_{Z}^{*}(t, z)=\widetilde{V_{E, Q}^{*}(t, z)}$.

Proof. Consider a point $\left(t_{0}, z_{0}\right)$ with $t_{0} \neq 0$. Let $\lambda_{0}:=\frac{z_{0}}{t_{0}}$.

Then, by Theorem 2.1,

$$
\varlimsup_{(t, z) \rightarrow\left(t_{0}, z_{0}\right)} H_{Z}(t, z)=\varlimsup_{\lambda \rightarrow \lambda_{0}} V_{E, Q}(\lambda)+\log \left|\lambda_{0}\right|
$$

and the right side is $\left.V_{E, Q}^{*} \widetilde{\left(t_{0},\right.} z_{0}\right)$ by $(2.7)$. This proves the result for $t \neq 0$ but since both sides (in the statement of Corollary 2.1) are PSH functions on $\mathbb{C}^{N+1}$ and agree for $t \neq 0$ they must agree on $\mathbb{C}^{N+1}$.

Note that the result in $(\underline{\mathrm{K}}$. Prop. 2.9.16]) is similar but not immediately applicable.

Corollaries 2.2, 2.3 and 2.4 deal with the convergence of sequences of pluricomplex Green functions for sequences of weights converging in various manners. Corollary 2.2 is proved, using a different method, in ([BL2, Lemma 7.3]).

Corollary 2.2. Let $E \subset \mathbb{C}^{N}$ be compact and $\left\{w_{j}\right\}_{j=1,2, \ldots}$ a sequence of admissible weights on $E$ and let $w$ also be an admissible weight on $E$. Suppose that $w_{j} \downarrow w$. Then

$$
\lim _{j} V_{E, Q_{j}}=V_{E, Q} .
$$

Proof. Let $Z_{j}:=Z_{j}\left(E, w_{j}\right)$ and $Z:=Z(E, w)$ be the associated circular sets in $\mathbb{C}^{N+1}$. Then, by hypothesis, the sets $\left\{Z_{j}(D)\right\}_{j=1,2, \cdots}$ (defined in (2.4)) are a decreasing family of compact sets whose intersection is $Z(D)$. By ([K, cor 5.1.2]) we have $\lim _{j} V_{Z_{j}(D)}=V_{Z(D)}$ for all $z \in \mathbb{C}^{N+1}$ and so $\lim _{j} H_{Z_{j}(D)}=H_{Z(D)}$. We conclude that $\lim _{j} H_{Z_{j}}=H_{Z}$ and using Theorem 2.1 the result follows.

Corollary 2.2'. Let $E \subset \mathbb{C}^{N}$ be compact and $\left\{E_{j}\right\}_{j=1,2, \cdots}$ be a decreasing family of compact sets in $\mathbb{C}^{N}$ with $\bigcap_{j=1}^{\infty} E_{j}=E$. For each $j=1,2, \cdots$ let $w_{j}$ be an admissible weight on $E_{j}$ and let $w$ be an admissible weight on $E$. Suppose that $w_{j} \downarrow w$ on $E$. Then $\lim _{j} V_{E_{j}, Q_{j}}=V_{E, Q}$ on $E$.

Proof. See the proof of Corollary 2.2.

Corollary 2.3. Let $E,\left\{w_{j}\right\}, w$ be as in Corollary 2.2, except that $w_{j} \downarrow w$ q.e. Then

$$
\lim _{j} V_{E, Q_{j}}^{*}=V_{E, Q}^{*} .
$$


Proof. Let $w_{j}^{\prime}=\operatorname{Inf}\left(w_{1}, \cdots, w_{j}\right)$ for $j=1,2, \cdots$ and $w^{\prime}=\operatorname{Inf}\left\{w_{j} \mid j=1,2, \cdots\right\}$. Then $w_{j}^{\prime} \downarrow w^{\prime}$ on $E$ and $w^{\prime}, w_{j}^{\prime}$ for $j=1,2, \cdots$ are admissible weights on $E$. It follows from Corollary 2.2 that

$$
\lim _{j} V_{E, Q_{j}^{\prime}}=V_{E, Q^{\prime}}
$$

However, by hypothesis, $w_{j}^{\prime}=w_{j}$ q.e. on $E$ (for $j=1,2, \cdots$ ) and $w^{\prime}=w$ q.e. on $E$. Thus by $\left(\left[\mathrm{BL2}\right.\right.$, Lemma 7.2]) we have $V_{E, Q_{j}}^{*}=V_{E, Q_{j}^{\prime}}^{*}$ for $j=1,2, \cdots$ and $V_{E, Q}^{*}=V_{E, Q^{\prime}}^{*}$

The result follows.

Corollary 2.4. Let $E,\left\{w_{j}\right\}, w$ be as in Corollary 2.2 except that $w_{j} \uparrow w$ q.e. Then $\lim _{j} V_{E, Q_{j}}^{*}=V_{E, Q}^{*}$.

Proof. There is a pluripolar set $P \subset E$ such that $w_{j} \uparrow w$ on $E \backslash P$. We let

$$
C(P)=\left\{(t, z) \in \mathbb{C}^{N+1} \mid \frac{z}{t} \in P\right\} .
$$

Then $C(P)$ is pluripolar in $\mathbb{C}^{N+1}$. Let $B$ be a large closed ball such that $B \supset Z(D)$. Then the family of compact sets $\left.\left\{Z_{j}(D) \cup C(P)\right) \cap B\right\}$ increases to $(Z(D) \cup C(P)) \cap B$ as $j \rightarrow+\infty$.

But each of the sets $\left(Z_{j}(D) \cup C(P)\right) \cap B$ differs from $Z_{j}(D)$ by a pluripolar set (similarly for $(Z(D) \cup C(P) \cap B)$ ). Thus by $([\mathrm{K}$, cor. 5.2.5 and 5.2.6])

$$
\begin{aligned}
& V_{Z_{j}(D)}^{*} \downarrow V_{Z(D)}^{*}, \\
& \text { so } \quad H_{Z_{j}}^{*} \downarrow H_{Z}^{*} \text {. }
\end{aligned}
$$

The result now follows from Corollary 2.1.

By Proposition 2.3, we may consider $L_{*}\left(\mu_{e q}(Z)\right)$, the push-forward of the equilibrium measure $\mu_{e q}(Z)$ under $L$. Since $\operatorname{supp}\left(\mu_{e q}(Z)\right) \subset \bar{Z} \subset \bigcup_{\lambda \in E} C_{\lambda}$ we have $\operatorname{supp}\left(L_{*}\left(\mu_{e q}(Z)\right) \subset E\right.$. There is however a more precise relation. The equilibrium measure on $Z$ and the weighted equilibrium measure on $E$ are related by:

Theorem 2.2. $L_{*}\left(\frac{1}{2 \pi} \mu_{e q}(Z)\right)=\mu_{e q}(E, w)$.

Proof. The proof is based on Lemma 3.3 in DeM], which itself is based on work of Briend. (Note that we use the convention of Klimek's book $[\mathrm{K}]$ for $d^{c}:=i(\bar{\partial}-\partial)$ not that of [DeM]. This results in the factor $\frac{1}{2 \pi}$ in the statement of Theorem 2.2.)

First we will prove the result in the case that $Z$ is regular.

$H_{Z}$ is continuous by Proposition 2.2 so, as a consequence of Theorem 2.1, $V_{E, Q}$ is continuous. Then

$$
\begin{gathered}
d d^{c} H_{Z}=d d^{c} L^{*}\left(V_{E, Q}\right)=L^{*}\left(d d^{c} V_{E, Q}\right) \text { for } t \neq 0 \text { and so } \\
\left(d d^{c} H_{Z}\right)^{N}=L^{*}\left(d d^{c} V_{E, Q}\right)^{N}=L^{*}\left(\mu_{e q}(E, w)\right) \text { for } t \neq 0 .
\end{gathered}
$$

Let $\phi$ be a smooth compactly supported function on $\mathbb{C}^{N+1} \backslash\{t=0\}$. Then

$$
\int_{\mathbb{C}^{N+1}} \phi d \mu_{e q}(Z)=\int_{\mathbb{C}^{N+1}} \phi\left(d d^{c} V_{Z}\right)^{N+1}=\int_{\mathbb{C}^{N+1}} V_{Z} d d^{c} \phi \wedge\left(d d^{c} V_{Z}\right)^{N} .
$$


Now, as $\epsilon \downarrow 0, \operatorname{Max}\left(H_{Z}, \epsilon\right)-\epsilon \uparrow V_{Z}$ uniformly on $C^{N}$. So

$$
\int_{\mathbb{C}^{N+1}} V_{Z} d d^{c} \phi \wedge\left(d d^{c} V_{Z}\right)^{N}=\lim _{\epsilon \rightarrow 0} \int_{\mathbb{C}^{N+1}}\left(\operatorname{Max}\left(H_{Z}, \epsilon\right)-\epsilon\right) d d^{c} \phi \wedge\left(d d^{c} V_{Z}\right)^{N} .
$$

Note that $\left\{z \in \mathbb{C}^{N+1} \mid \operatorname{Max}\left(H_{Z}, \epsilon\right)=\epsilon\right\}$ is a neighborhood of $\bar{Z}$ in $\mathbb{C}^{N+1}$ and if $\operatorname{Max}\left(H_{Z}(z), \epsilon\right)>\epsilon$, then $V_{Z}=H_{Z}$. So, in the expression on the right of (2.18) we may replace $V_{Z}$ by $H_{Z}$ to obtain

$$
\int_{\mathbb{C}^{N+1}} V_{Z} d d^{c} \phi \wedge\left(d d^{c} H_{Z}\right)^{N}=\int_{\mathbb{C}^{N+1}} V_{Z} d d^{c} \phi \wedge L^{*}\left(d d^{c} V_{E, Q}\right)^{N} .
$$

The integrands in (2.17), (2.18) and (2.19) all have compact support in $\mathbb{C}^{N+1} \backslash$ $\{t=0\}$, so the right side of (2.19) is equal to

$$
\int_{\mathbb{C}^{N}}\left(\int_{C_{\lambda}} V_{Z} d d^{c} \phi\right) d \mu_{e q}(E, w)=\int_{\mathbb{C}^{N}}\left(\int_{\mathbb{C}_{\lambda}} \phi d d^{c} V_{Z}\right) d \mu_{e q}(E, w) .
$$

For $\lambda \in E$, we let $m_{\lambda}$ be the Lebesgue measure on the circle $|t|=w(\lambda)$ in $C_{\lambda}$ normalized to have total mass 1 . Then $\frac{1}{2 \pi} d d^{c} V_{Z \mid C_{\lambda}}=d m_{\lambda}$.

The right side of (2.19) is thus equal to

$$
\frac{1}{2 \pi} \int_{\mathbb{C}^{N}}\left(\int_{C_{\lambda}} \phi d m_{\lambda}\right) d \mu_{e q}(E, w)
$$

which proves Theorem 2.2 in case $Z$ is regular.

Lemmas 2.2 and 2.3 below will be used to reduce the general case to that of $Z$ being regular.

Lemma 2.2. Let $E$ be compact in $\mathbb{C}^{N}$. Let $E_{\epsilon}:=\left\{z \in \mathbb{C}^{N} \mid \operatorname{dist}(z, E) \leq \epsilon\right\}$, where dist is Euclidean distance. Then $E_{\epsilon}$ is locally regular.

Proof. It is standard that $E_{\epsilon}$ is regular (see $[\mathrm{K}$, cor. 5.1.5]). A regular set is not, in general, locally regular however. In this case the proof that $E_{\epsilon}$ is regular also shows it is locally regular since for all $a \in E_{\epsilon}$ and $B_{1}$ a ball centered at $a$ there is another ball $B_{2}$ (not necessarily centered at $a$ ) such that $a \in B_{2} \subset B_{1} \cap E_{t}$.

Lemma 2.3. Let $E$ be compact in $\mathbb{C}^{N}$ and $w$ an admissible weight on $E$. Then there exists a decreasing sequence of locally regular compact sets $\left\{E_{j}\right\}_{j=1,2, \ldots}$ and admissible, continuous weights $w_{j}$ on $E_{j}$ such that $w_{j} \downarrow w$ on $E$ and $w_{j+1} \leq w_{j}$ on $E_{j+1}($ for all $j=1,2, \cdots)$.

Proof. Let $E_{j}=\left\{z \in \mathbb{C}^{N} \mid \operatorname{dist}(z, E) \leq \frac{1}{j}\right\}$. Let $\varphi_{j}$ be a decreasing sequence of continuous functions on $E$ with $\varphi_{j} \downarrow w$. Let $w_{j}^{\prime}$ be a continuous extension of $\varphi_{j}$ to $E_{j}$. We take $w_{j}=\operatorname{Inf}\left(w_{j}^{\prime}, \cdots, w_{j}^{\prime}\right)$.

Now we complete the proof of Theorem 2.2. Given $E, w$ take $\left\{E_{j}\right\}$ and $w_{j}$ as in Lemma 2.3. Let $Z_{j}:=Z_{j}\left(E_{j}, w_{j}\right)$. By Corollary $2.2^{\prime}, V_{E_{j}, Q_{j}} \uparrow V_{E, Q}$, so by ([K, Theorem 3.6.1]) $\mu_{e q}\left(E_{j}, w_{j}\right) \rightarrow \mu_{e q}(E, w)$ weak*. The sets $Z_{j}$ decrease to $Z=$ $Z(E, w)$, so $V_{Z_{j}} \uparrow V_{Z}$ (by [K], cor 5.1.2]). Again it follows that $\mu_{e q}\left(Z_{j}\right) \rightarrow \mu_{e q}(Z)$ 
weak*. Now, for each $j, V_{E_{j}, Q_{j}}$ is continuous, so $Z_{j}$ is regular. Applying Theorem 2.2 we have

$$
L_{*}\left(\frac{1}{2 \pi} \mu_{e q}\left(Z_{j}\right)\right)=\mu_{e q}\left(E_{j}, w_{j}\right) .
$$

Taking the limit of each side (as $j \rightarrow+\infty$ ) of the above equation gives the result.

\section{The Bernstein-Markov inequality}

Given a compact set $E \subset \mathbb{C}^{N}$ and a finite positive Borel measure $\mu$ on $E$, we say that $(E, \mu)$ satisfies the Bernstein-Markov (B-M) inequality if, for every $\epsilon>0$, there exists a constant $C=C(\epsilon)>0$ such that, for all holomorphic polynomials $p$ we have

$$
\|p\|_{E} \leq C(1+\epsilon)^{\operatorname{deg}(p)}\|p\|_{L^{2}(\mu)} .
$$

This inequality may be used to relate $L^{2}$ properties of polynomials with potentialtheoretic invariants of $E$ (see [B1] and BL1] for conditions under which the inequality holds).

We will introduce a "weighted" version of the B-M inequality.

Given a compact set $E \subset \mathbb{C}^{N}$, an admissible weight $w$ on $E$ and a finite positive Borel measure $\mu$ on $E$, we say that $(E, w, \mu)$ satisfies the weighted $B$ - $M$ inequality if for all $\epsilon>0$, there exists a constant $C=C(\epsilon)>0$ such that, for all weighted polynomials $w^{d} p$ we have

$$
\left\|w^{d} p\right\|_{E} \leq C(1+\epsilon)^{d}\left\|w^{d} p\right\|_{L^{2}(\mu)} .
$$

Of course, for $w \equiv 1,(3.2)$ reduces to $(3.1)$.

We will relate the weighted B-M inequality for $(E, w, \mu)$ to the B-M inequality on $\bar{Z}$ with respect to a certain associated measure $\nu$. The measure $\nu$ is defined as follows:

$$
\nu=m_{\lambda} \otimes \mu \text { for } \lambda \in E \text { so that } \operatorname{supp}(\nu) \subset \bigcup_{\lambda \in E} C_{\lambda} .
$$

Recall that $m_{\lambda}$ is the normalized Lebesgue measure on the circle $|t|=w(\lambda)$ in $C_{\lambda}$.

That is, for $\phi$ continuous with compact support in $\mathbb{C}^{N+1} \backslash\{t=0\}$ we have

$$
\int_{\mathbb{C}^{N+1}} \phi d \nu=\int_{E}\left(\int_{C_{\lambda}} \phi d m_{\lambda}\right) d \mu(\lambda) .
$$

Theorem 3.1. $(E, w, \mu)$ satisfies the weighted $B-M$ inequality if and only if $(\bar{Z}, \nu)$ satisfies the $B-M$ inequality.

Proof. First (using the notation of Lemma 2.1) we prove

Lemma 3.1. $\left\|P_{d}\right\|_{L^{2}(\nu)}=\left\|w^{d} G_{d}\right\|_{L^{2}(\mu)}$.

Proof. $P_{d}$ is a homogeneous polynomial of degree $d$ on $\mathbb{C}^{N+1}$. Now

$$
\begin{aligned}
\int_{\mathbb{C}^{N+1}}\left|P_{d}(t, z)\right|^{2} d \nu & =\int_{E}\left(\int_{C_{\lambda}}\left|P_{d}(t, z)\right|^{2} d m_{\lambda}\right) d \mu(\lambda) \\
& =\int_{E}\left|w^{d} G_{d}\right|^{2} d \mu(\lambda), \text { using (2.9) } \\
& =\left\|w^{d} G_{d}\right\|_{L^{2}(\mu)} .
\end{aligned}
$$


Now, suppose $(\bar{Z}, \nu)$ satisfies the B-M inequality. Applying that inequality to homogeneous polynomials, using Lemmas 2.1 and 3.1 we obtain the weighted B-M inequality for $(E, w, \mu)$.

For the converse, suppose $(E, w, \mu)$ satisfies the weighted B-M inequality.

We first note that if two monomials are of different degrees, they are orthogonal in $L^{2}(\nu)$ since their restrictions to any $C_{\lambda}$ are orthogonal in $L^{2}\left(m_{\lambda}\right)$. Hence for a polynomial $p$ on $\mathbb{C}^{N+1}$, written as a sum of homogeneous polynomials,

$$
p=\sum_{i=0}^{d} p_{i}
$$

we have

$$
\|p\|_{L^{2}(\nu)}=\sum_{i=0}^{d}\left\|p_{i}\right\|_{L^{2}(\nu)}
$$

For any $\epsilon>0$ there is a $C>0$ such that

$$
\|p\|_{Z} \leq \sum_{i=0}^{d}\left\|p_{i}\right\|_{Z} \leq C(1+\epsilon)^{d}\left\|p_{i}\right\|_{L^{2}(\nu)} \leq C(d+1)(1+\epsilon)^{d}\|p\|_{L^{2}(\nu)},
$$

where the second inequality comes from Lemmas 2.1, 3.1 and the weighted B-M inequality for $(E, w, \mu)$. The third inequality in (3.7) comes from (3.6). The B-M inequality for $(\bar{Z}, \nu)$ follows from $(3.7)$.

Corollary 3.1. Suppose $\bar{Z}$ is regular. Then $\left(E, w, \mu_{e q}(E, w)\right)$ satisfies the weighted $B-M$ inequality.

Proof. It is a result of Nguyen-Zeriahi $[\mathrm{NZ}$ ] combined with ([K, Corollary 5.6.7]) that $\left(\bar{Z}, \mu_{e q}(\bar{Z})\right)$ satisfies the B-M inequality. However, by Theorem $2.2, \frac{1}{2 \pi} \mu_{e q}(\bar{Z})=$ $m_{\lambda} \otimes \mu_{e q}(E, w)$, so applying Theorem 3.1, the result follows.

We will give another general situation in which the weighted B-M inequality holds (see also [StTo, Theorem 3.2.3 (vi)).

Theorem 3.2. Let $E$ be a compact set $\subset \mathbb{R}^{N}$ and let $w$ be continuous on $E$ with $\inf _{z \epsilon E} w(z)>0$. Suppose that $\sigma$ is a finite positive Borel measure on $E$ and $(E, \sigma)$ satisfies the $B-M$ inequality. Then $(E, w, \sigma)$ satisfies the weighted $B-M$ inequality.

Proof. $\log w$ is continuous on $E$ and so may be, by the Weierstrass theorem, approximated by (real) polynomials. That is, given $\epsilon>0$ there exists $g_{\epsilon}=g_{\epsilon}\left(x_{1}, \cdot, x_{n}\right)$, a real polynomial, such that $\left\|\log w-g_{\epsilon}\right\|_{E} \leq \epsilon$. Taking exponentials, we have

$$
e^{-\epsilon} \leq \frac{w}{\exp \left(g_{\epsilon}\right)} \leq e^{\epsilon} \quad \text { for } z \in E
$$

We consider $g_{\epsilon}$ as a holomorphic polynomial

$$
g_{\epsilon}=g_{\epsilon}\left(z_{1}, \cdots, z_{N}\right) .
$$

Taking sufficently many terms in the power series for $\exp \left(g_{\epsilon}\right)$ we get a holomorphic polynomial $H$ such that, for $\epsilon$ sufficiently small,

$$
1-2 \epsilon \leq \frac{w}{H} \leq 1+2 \epsilon \quad \text { for } \quad z \in E .
$$

Now, consider a weighted polynomial $w^{d} G$ and let

$$
J:=G H^{d} \text {. }
$$


Then

$$
\begin{gathered}
w^{d}|G|=|J|\left(\frac{w}{|H|}\right)^{d} \quad \text { so that } \\
|J|(1-2 \epsilon)^{d} \leq w^{d}|G| \leq|J|(1+2 \epsilon)^{d} \text { for } z \in E .
\end{gathered}
$$

By the B-M inequality for $(E, \sigma)$ we have, given $\epsilon_{1}>0$, a constant $C_{1}>0$ such that

$$
\|J\|_{E} \leq C_{1}\left(1+\epsilon_{1}\right)^{(h+1) d}\|J\|_{L^{2}(\sigma)},
$$

where $h:=\operatorname{deg} H$. Hence

$$
\begin{aligned}
\left\|w^{d} G\right\|_{E} & \leq\|J\|_{E}(1+2 \epsilon)^{d} \quad \text { by the right inequality in (3.13) } \\
& \leq C_{1}\left(1+\epsilon_{1}\right)^{(h+1) d}(1+2 \epsilon)^{d}\|J\|_{L^{2}(\sigma)} \quad \text { by }(3.14) \\
& \leq C_{1}\left(1+\epsilon_{1}\right)^{(h+1) d} \frac{(1+2 \epsilon)^{d}}{(1-2 \epsilon)^{d}}\left\|w^{d} G\right\|_{L^{2}(\sigma)}
\end{aligned}
$$

by the left inequality in (3.13).

Now, $\epsilon>0$ having been chosen, and $h$ fixed, we choose $\epsilon_{1}$ so that $\left(1+\epsilon_{1}\right)^{(h+1)} \leq$ $1+\epsilon$ and so we obtain

$$
\left\|w^{d} G\right\|_{E} \leq C_{1} \frac{(1+\epsilon)^{d}(1+2 \epsilon)^{d}}{(1-2 \epsilon)^{d}}\left\|w^{d} G\right\|_{L^{2}(\sigma)} .
$$

But $\epsilon>0$ is arbitrary, so the weighted B-M inequality holds.

Example 3.1. Let $B_{R}=\left\{x \in \mathbb{R}^{N}|| x \mid \leq R\right\}$ be the (real) ball of radius $R$ (center the origin). Then $\left(B_{R}, d x\right)$ satisfies the B-M inequality (see [B1]), where $d x$ denotes Lebesgue measure.

Let $w(x)$ be any continuous positive function on $B_{R}$. Then by Theorem 3.2, $\left(B_{R}, w, d x\right)$ satisfies the weighted B-M inequality.

\section{4. $L^{2}$ THEORY OF WEIGHTED POLYNOMIALS}

Let $E$ be a compact non-pluripolar subset of $\mathbb{C}^{N}, w$ an admissible weight on $E$, and $\mu$ a finite positive Borel measure with $\operatorname{supp}(\mu)=E$. For $d$ a positive integer, the monomials are linearly independent in $L^{2}\left(w^{2 d} \mu\right)$ (B1, Prop. 3.5] adapts to this situation). Ordering via a lexicographic ordering (which respects total ordering) on their multi-index exponents and applying the Gram-Schmidt procedure we obtain orthonormal polynomials $\left\{p_{\alpha}^{d}(z, \mu)\right\}_{\alpha \in \mathbb{N}^{n}}$. They satisfy

$$
\int_{\mathbb{C}^{N}} p_{\alpha}^{d}(z, \mu) p_{\beta}^{d}(z, \mu) w^{2 d} d \mu=\delta_{\alpha \beta}
$$

for $\alpha, \beta$ multi-indices.

We can write

(4.2) $p_{\alpha}^{d}(z, \mu)=a_{\alpha}^{d} z^{\alpha}+$ (monomials of lower lexicographic order) where $a_{\alpha}^{d}>0$.

We will only consider these polynomials where $|\alpha|=d$.

In the case that $(E, w, \mu)$ satisfies the weighted B-M inequality we will show that the leading exponents $\left\{a_{\alpha}^{d}\right\}$ have asymptotic limits in the following sense. First we let

$$
\Sigma_{0}:=\left\{\theta \in \mathbb{R}^{N} \mid \theta=\left(\theta_{1}, \cdots, \theta_{N}\right), \sum_{j=1}^{n} \theta_{j}=1, \theta_{j}>0\right\} .
$$


We consider sequences of multi-indices $\{\alpha(j)\}_{j=1,2, \ldots}$ with, for some $\theta \in \Sigma_{0}$,

$$
\lim _{j}|\alpha(j)|=+\infty \text { and } \lim _{j} \frac{\alpha(j)}{|\alpha(j)|}=\theta .
$$

Condition (4.4) implies that $d=d(j)=|\alpha(j)| \rightarrow+\infty$.

Theorem 4.1. Suppose $(E, w, \mu)$ satisfies the weighted B-M inequality and $\{\alpha(j)\}$ is a sequence of multi-indices satisfying (4.4). Then

$$
\lim _{j}\left(a_{\alpha(j)}^{d}\right)^{\frac{1}{d}}=\frac{1}{\tau^{w}(E, \theta)},
$$

where $\tau^{w}(E, \theta)$ is the weighted directional Tchebyshev constant of $E$ in the direction $\theta$.

Proof. First, we recall the definition of a weighted directional Tchebyshev constant (see [BL2]). For $\alpha$ a multi-index we let $\mathcal{P}(\alpha)=\left\{q \mid q=z^{\alpha}+\sum_{\beta<\alpha} c_{\beta} z^{\beta}\right\}$, where $c_{\beta} \in \mathbb{C}$ and the notation $\beta<\alpha$ is used to denote the fact that the multi-index $\beta$ precedes $\alpha$ in the lexicographic ordering on the multi-indices.

For $\alpha$ a multi-index with $|\alpha|=d$ we let $t_{\alpha}^{d}$ denote a (Tchebyshev) polynomial which minimizes $\left\{\left\|w^{d} q\right\|_{E} \mid q \in \mathcal{P}(\alpha)\right\}$. That is, $t_{\alpha}^{d} \in \mathcal{P}(\alpha)$ and

$$
\left\|w^{d} t_{\alpha}^{d}\right\|_{E}=\inf \left\{\left\|w^{d} q\right\|_{E} \mid q \in \mathcal{P}(\alpha)\right\} .
$$

Then (see [BL2]) it is known that for a sequence of multi-indices $\{\alpha(j)\}_{j=1,2, \cdots}$ satisfying (4.4) the limit

$$
\tau^{w}(E, \theta):=\lim _{j}\left\|w^{d} t_{\alpha(j)}^{d}\right\|_{E}^{\frac{1}{d}}
$$

exists and is called the weighted Tchebyshev constant in the direction $\theta \in \Sigma_{0}$.

It follows from general Hilbert space theory that

$$
a_{\alpha}^{d}=\frac{1}{\left\|w^{d} q_{\alpha}^{d}\right\|_{L^{2}(\mu)}},
$$

where $q_{\alpha}^{d}$ is the unique polynomial in $\mathcal{P}(\alpha)$ satisfying

$$
\left\|w^{d} q_{\alpha}^{d}\right\|_{L^{2}(\mu)}=\inf \left\{\left\|w^{d} q\right\|_{L^{2}(\mu)} \mid q \in \mathcal{P}(\alpha)\right\} .
$$

Given $\epsilon>0$, there is a constant $C>0$ such that

$$
\begin{aligned}
\left\|w^{d} q_{\alpha}^{d}\right\|_{E} & \leq C(1+\epsilon)^{d}\left\|w^{d} q_{\alpha}^{d}\right\|_{L^{2}(\mu)} \quad \text { by the weighted B-M inequality } \\
& \leq C(1+\epsilon)^{d}\left\|w^{d} t_{\alpha}^{d}\right\|_{L^{2}(\mu)} \quad \text { by } \\
& \leq C_{1}(1+\epsilon)^{d}\left\|w^{d} t_{\alpha}^{d}\right\|_{E}
\end{aligned}
$$

since the $L^{2}$-norm is bounded by a constant times the sup norm for a finite positive measure with compact support.

Hence, for every $\epsilon>0$ there is a constant $C_{1}>0$ such that

$$
\left\|w^{d} t_{\alpha}^{d}\right\|_{E} \leq\left\|w^{d} q_{\alpha}^{d}\right\|_{E} \leq C_{1}(1+\epsilon)^{d}\left\|w^{d} t_{\alpha}^{d}\right\|_{E} .
$$

Now, given a sequence of multi-indices $\{\alpha(j)\}$ satisfying (4.4), taking the $1 / d$ powers of the expressions in (4.10), letting $j \rightarrow \infty$, using (4.6), (4.7), (4.9) and the fact that $\epsilon>0$ is arbitrary, the result follows. 
On $\mathbb{R}^{N}$ we consider orthonormal polynomials with respect to the inner product given by $e^{-H(x)} d x$, where $d x$ is Lebesgue measure on $\mathbb{R}^{N}$ and $H(x)$ satisfies

i) $H(x)$ is homogeneous of degree $\gamma>0$. That is,

$$
\begin{array}{r}
H(c x)=c^{\gamma} H(x) \quad c \in \mathbb{R} . \\
\text { ii) } H(x)>0 \text { for all } x \neq 0 .
\end{array}
$$

We let $\left\{p_{\alpha}(x)\right\}_{\alpha \in \mathbb{N}^{N}}$ denote the orthonormal polynomials obtained by applying the Gram-Schmidt procedure to the (real) monomials ordered via a lexicographic ordering (respecting total order) of their exponents. Then

$$
\int_{\mathbb{R}^{N}} p_{\alpha}(x) p_{\beta}(x) e^{-H(x)} d x=\delta_{\alpha \beta}
$$

for any two multi-indices $\alpha, \beta$.

We write

$$
p_{\alpha}(x)=a_{\alpha} x^{\alpha}+\text { (sum of monomials of lower lexicographic order) where } a_{\alpha}>0 \text {. }
$$

We will obtain asymptotic estimates (see Theorem 4.2) for $\left|a_{\alpha}\right|^{\frac{1}{\alpha \mid}}$ for a sequence of multi-indices satisfying (4.4). In the case $N=1$, these estimates are Theorem VII, 1.2 of [SaTo]. In that case explicit knowledge of the set $S_{w}$ (defined below) yields an explicit form to the right hand side of (4.24). It would be of interest to find $S_{w}$ explicitly in the case $N>1$.

In the one-dimensional case $(N=1)$ this gives a version of the so-called weak asymptotics and in this case considerably more detailed asymptotic results are known (see [SaTo] or Dei]).

Theorem 4.2. Let $H(x)$ be as in (4.11) and $a_{\alpha}$ for $\alpha \in \mathbb{N}^{N}$ the leading coefficients of the orthonormal polynomial $p_{\alpha}(x)$ (as in (4.13)). Let $w$ be the weight function $e^{-\frac{H(x)}{2}}$ on $\mathbb{R}^{N}$ and $V_{\mathbb{R}^{N}, Q}$ the associated weighted pluricomplex Green function. Let $S_{w}:=\operatorname{supp}\left(d d^{c} V_{\mathbb{R}^{N}, Q}\right)^{N}$. Let $\{\alpha(j)\}_{j=1,2, \cdots}$ be a sequence of multi-indices satisfying (4.4). Then

$$
\lim _{j} a_{\alpha(j)}^{\frac{1}{d}} d^{\frac{1}{\gamma}}=\frac{1}{\tau^{w}\left(S_{w}, \theta\right)} .
$$

Proof. From general Hilbert space theory,

$$
a_{\alpha}^{-1}=\inf \left\{\left\|e^{-\frac{H(x)}{2}} q(x)\right\|_{L^{2}\left(\mathbb{R}^{N}\right)} \mid q \in \mathcal{P}_{\mathbb{R}}(\alpha)\right\},
$$

where $\mathcal{P}_{\mathbb{R}}(\alpha)=\left\{\right.$ polynomials of the form $x^{\alpha}+\sum_{\beta<\alpha} r_{\beta} x^{\beta}$ with $r_{\beta} \in \mathbb{R}$.

For $|\alpha|=d$ we scale by $x=d^{\frac{1}{\gamma}} y$. We get

$$
a_{\alpha}^{-1}=d^{\frac{d}{\gamma}} \inf \left\{\left\|e^{-\frac{d H(y)}{2}} q(y)\right\|_{L^{2}\left(\mathbb{R}^{N}\right)} \mid q \in \mathcal{P}_{R}(\alpha)\right\} .
$$

Consider the weight $w(y)=e^{-\frac{H(y)}{2}}$ on $\mathbb{R}^{N} \subset \mathbb{C}^{N}$. This weight is admissible. We let $Q(y)=\frac{H(y)}{2}$. It is known $\left(\left[\underline{\mathrm{SaTo}}\right.\right.$, appendix B) that $S_{w}:=\left(d d^{c} V_{\mathbb{R}^{N}, Q}\right)^{N}$ has compact support. For any weighted polynomial $w^{d} p$ we have

$$
\left|w^{d} p(y)\right| \leq\left\|w^{d} p\right\|_{S_{w}} \exp \left(d\left(V_{\mathbb{R}^{N}, Q}-Q\right)\right) .
$$


In particular

$$
\sup _{\mathbb{R}^{N}}\left|w^{d} p\right|=\sup _{S_{w}}\left|w^{d} p\right| .
$$

Now $V_{\mathbb{R}^{N}, Q} \in \mathcal{L}$ so, fixing $R>0$ large, using (4.16), there is a constant $A>0$ such that

$$
\left|w^{d} p(y)\right| \leq\left\|w^{d} p\right\|_{S_{w}} e^{-A d|y|^{\gamma}} \text { for }|y| \geq R .
$$

Hence, using (4.18),

$$
\left\|w^{d} p\right\|_{L^{2}\left(\mathbb{R}^{N}\right)}^{2} \leq\left\|w^{d} p\right\|_{L^{2}\left(B_{R}\right)}^{2}+\left\|w^{d} p\right\|_{S_{w}}^{2} \int_{|y| \geq R} e^{-2 A d|y|^{\gamma}} d y .
$$

We may assume $S_{w} \subset B_{R}$. Then

$$
\begin{aligned}
& \left\|w^{d} p\right\|_{S_{w}}=\left\|w^{d} p\right\|_{B_{R}} \leq C(1+\epsilon)^{d}\left\|w^{d} p\right\|_{L^{2}\left(B_{R}\right)} \text { since } \\
& \left.\left(B_{R}, w, d x\right) \text { satisfies the weighted B-M inequality (see Example } 3.1\right) .
\end{aligned}
$$

Now simple estimates show there is a constant $c_{1}>0$ such that

$$
\int_{|y| \geq R} e^{-2 A d|y|^{\gamma}} d y \leq e^{-d c_{1}}
$$

We get

$$
\left\|w^{d} p\right\|_{L^{2}\left(\mathbb{R}^{N}\right)} \leq\left\|w^{d} p\right\|_{L^{2}\left(B_{R}\right)}\left(1+\frac{C^{2}(1+\epsilon)^{2 d}}{e^{d c_{1}}}\right)^{\frac{1}{2}} .
$$

However for $\epsilon>0$ sufficiently small, the expression on the right of (4.22) is bounded in $d$. It follows that for a sequence of multi-indices $\{\alpha(j)\}$ satisfying (4.4),

$\lim _{j \rightarrow \infty}\left[\inf \left\{\left\|w^{d} q\right\|_{L^{2}\left(\mathbb{R}^{N}\right)} \mid q \in \mathcal{P}_{\mathbb{R}}(\alpha(j))\right\}^{\frac{1}{d}}\right]=\lim _{j \rightarrow \infty}\left[\inf \left\{\left\|w^{d} q\right\|_{L^{2}\left(B_{R}\right)} \mid q \in \mathcal{P}_{\mathbb{R}}(\alpha(j))\right\}^{\frac{1}{d}}\right]$.

But, by (the proof of) Theorem 4.1 the limit on the right side of (4.23) exists and, using (4.17), it may be identified with $\tau^{w}\left(S_{w}, \theta\right)$ using (4.1). Hence we obtain

$$
\lim _{j \rightarrow \infty} a_{\alpha(j)}^{\frac{1}{d}} d^{\frac{1}{\gamma}}=\frac{1}{\tau^{w}\left(S_{w}, \theta\right)} .
$$

\section{General Circular Sets}

The circular sets which arise in the form $\hat{\bar{Z}}(E, w)$ (i.e. the polynomially convex hull of a set of the form $Z(E, w))$ are

i) polynomially convex,

ii) circular,

iii) compact,

iv) non-pluripolar.

However, they are not the most general sets with the Properties i), ii), iii), iv). We will show, however, that the most general set with those properties is an increasing union of sets of the form $\hat{\bar{Z}}(E, w)$. Propositions 5.3 and 5.4 show that equilibrium measures and Theorem 2.2 are compatible with this limiting procedure. 
Let $Z \subset \mathbb{C}^{N+1}$ be a set with Properties i), ii), iii), iv) above. Then the origin is an interior point of $Z$. We associate to $Z$ the function on $\mathbb{C}^{N}$ defined by

$$
w(\lambda):=\sup \left\{|t| \mid(t, z) \in Z \cap C_{\lambda}\right\} .
$$

Then $w(\lambda)>0$ for all $\lambda$ and $w$ is bounded above. We let $Q(\lambda):=-\log w(\lambda)$.

Proposition 5.1. $w$ is u.s.c. on $\mathbb{C}^{N}$.

Proof. Fix $\lambda^{0} \in \mathbb{C}^{N}$. Let $\left\{\lambda^{s}\right\}_{s=1,2, \cdots}$ be a sequence in $\mathbb{C}^{N}$ converging to $\lambda^{0}$. We may suppose, passing to a subsequence if necessary, that $\lim _{s}\left(\lambda^{s}\right):=w^{0}$ exists. The points $\left(w\left(\lambda^{s}\right), \lambda_{1}^{s} w\left(\lambda^{s}\right), \cdots, \lambda_{N}^{s} w\left(\lambda^{s}\right)\right) \in Z \cap C_{\lambda^{s}}$ so, since $Z$ is compact, the point $\left(w^{0}, \lambda_{1}^{0} w^{0}, \cdots, \lambda_{N}^{0} w^{0}\right) \in Z \cap C_{\lambda^{0}}$. Thus, by definition of $w, w\left(\lambda^{0}\right) \geq w^{0}=$ $\lim _{s} w\left(\lambda^{s}\right)$.

Proposition 5.2. $Q^{*}$ is $P S H$ on $\mathbb{C}^{N}$.

Proof. $Z=\left\{(t, z) \in \mathbb{C}^{N+1} \mid H_{Z}(t, z) \leq 0\right\}$.

Now

$$
\begin{aligned}
H_{Z}(t, z) & =\log |t|+H_{Z}(1, \lambda) \text { so that } \\
\log w(\lambda) & =-H_{Z}(1, \lambda) \text { and } \\
Q(\lambda) & =H_{Z}(1, \lambda) .
\end{aligned}
$$

But $H_{Z}=H_{Z}^{*}$ outside a circular pluripolar set in $\mathbb{C}^{N+1}$, so by BL2, Lemma 6.1], $H_{Z}(1, \lambda)=H_{Z}^{*}(1, \lambda)$ q.e. on $\mathbb{C}^{N}$.

Thus $Q^{*}=H_{Z}^{*}(1, \lambda)$.

Example 5.1. $Z=\left\{|t|^{2}+\left|z_{1}\right|^{2}+\cdots+\left|z_{N}\right|^{2} \leq 1\right\} \subset \mathbb{C}^{N+1}$. Then $w(\lambda)=$ $\left(1+\left|\lambda_{1}\right|^{2}+\cdots+\left|\lambda_{N}\right|^{2}\right)^{-\frac{1}{2}}$ and $Q(\lambda)=\frac{1}{2} \log \left(1+\left|\lambda_{1}\right|^{2}+\cdots+\left|\lambda_{N}\right|^{2}\right)$.

As the above example illustrates, in general the functions $w(\lambda)$ which arise in this way are not admissible weights. In particular, $\lim _{|\lambda| \rightarrow \infty}|\lambda| w(\lambda) \neq 0$.

Let $Z_{R}:=\left\{(t, z) \in Z \mid \frac{|z|}{|t|} \leq R\right\}$.

Propostion 5.3. $\lim _{R \rightarrow \infty} \mu_{e q}\left(Z_{R}\right)=\mu_{e q}(Z)$ weak* .

Proof. The family of sets $Z_{R} \cup T$ increases to $Z \cup T$ (as $R \rightarrow \infty$ ), where $T$ is the pluripolar set $\left\{(t, z) \in \mathbb{C}^{N+1} \mid t=0\right\}$.

From $\left(\left[\mathrm{K}\right.\right.$, cor 5.2.5 and 5.2.6]) it follows that $V_{Z_{R}}^{*} \downarrow V_{Z}^{*}$ as $R \rightarrow \infty$. The result follows from the continuity of the Monge-Ampère operator under decreasing limits K.

Proposition 5.4. $\lim _{R \rightarrow \infty} L_{*}\left(\frac{1}{2 \pi} \mu_{e q}\left(Z_{R}\right)\right)=\left(d d^{c} Q^{*}\right)^{N}$.

Proof. Let $w_{R}=\left.w\right|_{B(0, R)}$. Let $Q_{R}=-\log w_{R}$. Then the family of PSH functions $V_{B(0, R), Q_{R}}^{*}$ is decreasing as $R \rightarrow \infty$. Since $Q^{*}$ is PSH, $V_{B(0, R), Q_{R}}^{*}=Q^{*}$ on $B(0, R)$, so $V_{B(0, R), Q_{R}}^{*} \downarrow Q^{*}$. The result follows using Theorem 2.2.

\section{REFERENCES}

[A] H. Alexander, Projective Capacity. In Recent Developments in Several Complex Variables, Ann. of Math. Studies 100 (1981), 3-27. MR627747 (82k:32014)

[B1] T. Bloom, Orthogonal polynomials in $\mathbb{C}^{n}$, Indiana University Math. J. 46 no. 2 (1997), 427-452. MR:1481598 (98j:32006) 
[B2] T. Bloom, Random polynomials and Green functions, Int. Math. Res. Not. 28 (2005), 1689-1708. MR2172337 (2006k:32069)

[BL1] T. Bloom and N. Levenberg, Capacity convergence results and applications to a BernsteinMarkov inequality, Trans. Amer. Math. Soc. 351 no. 12 (1999), 4753-4767. MR1695017 (2000c:32087)

[BL2] T. Bloom and N. Levenberg, Weighted pluripotential theory on $\mathbb{C}^{N}$, Amer. J. of Math. 125 (2003), 57-103. MR1953518 (2003k:32045)

[BLM] T. Bloom, N. Levenberg and S. Ma'u, Robin functions and extremal functions, Ann. Polon. Math. 80 (2003), 55-84. MR1972834(2004i:31010)

[Dei] P. Deift, Orthogonal Polynomials and Random Matrices: A Riemann-Hilbert Approach, Courant Lecture Notes, Amer. Math Society 3 (1999). MR1677884 (2000g:47048)

[DeM] L. DeMarco, Dynamics of rational amps, Lyapunov exponents, bifunctions and capacity, Math. Ann. 326 (2003), 43-73. MR.1981611 (2004f:32044)

$[\mathrm{J}] \quad$ M. Jedrzejowski, The homogeneous transfinite diameter of a compact set in $\mathbb{C}^{N}$, Ann. Polon. Math. 55 (1991), 191-205. MR:1141434(92m:31009)

[K] M. Klimek, Pluripotential Theory, London Mathematical Society Monographs, New Series \#6, Oxford University Press, 1991. MR.1150978 (93h:32021)

[Si1] J. Siciak, Extremal plurisubharmonic functions in $\mathbb{C}^{N}$, Ann. Polon. Math. 39 (1981), 175-211. MR617459 (83e:32018)

[Si2] J. Siciak, On series of homogeneous polynomials and their partial sums, Ann. Polon. Math. 51 (1991), 289-302. MR1094001 (92d:32004)

[Si3] J. Siciak, A remark on Tchebysheff polynomials in $\mathbb{C}^{N}$, Univ. Iagel. Acta Math. 35 (1997), 37-45. MR1458043 (98d:32019)

[SaTo] E.B. Saff and V. Totik, Logarithmic Potentials with External Fields, Grundlehren Math. Wiss. [Fundamental Principles of Mathematical Sciences] 316 (1997). MR1485778 (99h:31001)

[StTo] H. Stahl and V. Totik, General Orthogonal Polynomials, Encyclopedia of Mathematics and its Application, vol. 43, Cambridge University Press, 1992. MR 1163828 (93d:42029)

[NZ] T.V. Nguyen and A. Zeriahi, Familles de polynômes presques partout bornées, Bull. Soc. Math. Fr. 107 (1983), 81-91. MR699992 (85b:32026)

Department of Mathematics, University of Toronto, Toronto, Ontario, Canada M5S 3G3

E-mail address: bloom@math.utoronto.ca 